\title{
A new family of rotation-covariant wavelets on the hexagonal lattice
}

\author{
Laurent Condat $^{a}$, Brigitte Forster-Heinlein ${ }^{a}$ and Dimitri Van De Ville ${ }^{b}$ \\ ${ }^{a}$ GSF - National Research Center for Environment and Health, 85764 Neuherberg, Germany \\ ${ }^{b}$ Biomedical Imaging Group (BIG), EPFL, CH-1015 Lausanne, Switzerland
}

\begin{abstract}
We present multiresolution spaces of complex rotation-covariant functions, deployed on the 2-D hexagonal lattice. The designed wavelets, which are complex-valued, provide an important phase information for image analysis, which is missing in the discrete wavelet transform with real wavelets. Moreover, the hexagonal lattice allows to build wavelets having a more isotropic magnitude than on the Cartesian lattice. The associated filters, fully characterized in the Fourier domain, yield an efficient FFT-based implementation.
\end{abstract}

Keywords: Complex wavelets, 2-D lattices, hexagonal sampling, multiresolution, scaling functions, rotationcovariance

\section{INTRODUCTION}

Multiresolution analysis and the discrete wavelet transform (DWT) have proved to form a powerful framework in a wide range of signal processing applications. When applied to 2-D signals and images defined on the traditional Cartesian lattice, the most frequently used DWT are separable; their basis functions and filters are simply tensor products of the 1-D ones, which makes the implementation simple. The downside, however, is that these decompositions tend to privilege the vertical and horizontal directions. They also create a "diagonal" cross-term that does not have a straightforward directional interpretation. So, the need exists for transforms able to correct this deficiency of the separable transforms and to capture the regularity of 1-D singularities having arbitrary orientations, like edges in the images. Non-separable wavelets, by contrast, offer more freedom and can be better tuned to provide more isotropy. To this end, on the Cartesian lattice, the quincunx subsampling scheme can advantageously replace the dyadic scheme. ${ }^{1,2}$

In this work, we depart from the Cartesian lattice $\mathbb{Z}^{2}$ and propose multiresolution bases on the hexagonal lattice. Hexagonal sampling is well known to present superior properties in many domains: its twelve fold symmetry implies more isotropy and makes its geometrical and topological characteristics better than those of the Cartesian lattice. It is also known that hexagonal sampling allows to represent isotropic band-limited functions with a lesser sampling density on the hexagonal lattice than on the Cartesian one. ${ }^{3}$ Hexagonal multiresolution analyses have a large area of potential applications, since it is possible to convert a classical image, defined on the Cartesian lattice, onto the hexagonal lattice, where the processing using the DWT may be performed. We proposed recently a new conversion method between these two lattices for this purpose. ${ }^{4}$ It has the appealing property of being fully reversible; so, the Cartesian to hexagonal step does not lose any information. Therefore, the challenge is to design wavelet bases that fully exploit the superior properties of the hexagonal lattice.

When designing non-separable wavelets, one possibility is to concentrate on the design of the filters only. Indeed, applying the DWT to an image is equivalent to feeding it to a filterbank, that splits it into one lowpass and several highpass subsampled channels. ${ }^{5}$ The lifting technique provides a powerful framework for designing such multidimensional filterbanks; ${ }^{6}$ the wavelets are calculated afterwards from the filter coefficients by iterative methods. However, this often results in wavelets with unfavorable properties, e.g. fractal-like functions. ${ }^{7}$ In this

Further author information: (Send correspondence to L.C.)

L.C.: E-mail: laurent.condat@gsf.de, Telephone: +49 8928918322

B.F: E-mail: brigitte.forster@gsf.de, Telephone: +49 8931872349

D.V.V.: E-mail: dimitri.vandeville@epfl.ch, Telephone: +41 216935142 
work, we design the scaling functions and wavelets directly, with explicit formulas in the Fourier domain - an approach that is often favored by mathematicians. ${ }^{8}$ The filters then follow directly.

Besides good isotropic property, the ability of an image transform to identify the local geometric coherence of structures like edges, ridges, or corners, is at the heart of image analysis. The best way to combine isotropy of the magnitude (the detection of a feature should not depend on its orientation) with an angle information is to build a redundant transform with complex-valued wavelets. The amplitude-phase representation provides great robustness to image processing algorithms. To this end, a new family of complex multiresolution analyses has been proposed recently on the Cartesian lattice. ${ }^{9}$ So, the analysis of an image provides complex wavelet coefficients, whose magnitude can be used like with classical real DWTs, and phase encodes the local orientation of the feature. The price to pay for this additional phase information is the $2 \times$-redundancy of the representation, since a real-valued image provides complex-valued coefficients. An alternative approach of the literature, that is increasingly popular, is the dual-tree DWT: two real DWTs are developed in parallel, with filters forming approximate Hilbert pairs. ${ }^{10,11}$ This yields a better directionality than the DWT, at the expense of a $4 \times$ redundancy in 2-D. Moreover, the angle selectivity of the dual-tree DWT is limited to a set of finite orientations, while our approach is fully steerable. Finally, complex non-redundant DWT have been proposed, but they essentially amount to re-organize real wavelet coefficients into complex ones, so that the gain of the phase is made at the expense of a loss in resolution, with the finest wavelet band having resolution 3 or 4 times lesser than the one of the initial image. ${ }^{12-14}$ Our initial goal was to develop a non-redundant complex DWT on the

hexagonal lattice in the same spirit, but we found the redundant design, presented in this article, to have much better theoretical properties.

The paper is organized as follows. In Sect. 2, we recall some basics for the design of multi-dimensional wavelets. We present our new rotation-covariant B-splines in Sect. 3 and use them to build multiresolution analyses in Sect. 4. Then, in Sect. 5, we design the associated wavelet functions. Implementational aspects are discussed in Sect. 6.

\section{MULTIRESOLUTION ANALYSES ON THE HEXAGONAL LATTICE}

The hexagonal lattice (of the so-called first type, ${ }^{15}$ with normalized sampling density equal to one) $\Lambda_{\text {hex }}=$ $\mathbf{R}_{\text {hex }} \mathbb{Z}^{2}=\left\{\mathbf{R}_{\text {hex }} \mathbf{k} \mid \mathbf{k} \in \mathbb{Z}^{2}\right\}$ is characterized by its matrix

$$
\mathbf{R}_{\text {hex }}=\left[\begin{array}{ll}
\mathbf{r}_{1} & \mathbf{r}_{2}
\end{array}\right]=\sqrt{\frac{2}{\sqrt{3}}}\left[\begin{array}{cc}
1 & 1 / 2 \\
0 & \sqrt{3} / 2
\end{array}\right] .
$$

In order to define functions on the hexagonal lattice having multi-scale properties, one needs to define a $2 \times 2$ dilation matrix A, satisfying the following properties:

(1) The dilated lattice $\mathbf{A} \Lambda_{\text {hex }}$ forms a sublattice of the lattice $\Lambda_{\text {hex }}$, i.e., $\mathbf{A} \Lambda_{\text {hex }} \subset \Lambda_{\text {hex }}$. Equivalently, $\mathbf{A} \mathbf{R}_{\text {hex }}=\mathbf{R}_{\text {hex }} \mathbf{D}$ with $\mathbf{D}$ only having integer entries.

(2) The magnitude of each eigenvalue of $\mathbf{A}$ must be strictly larger than 1 to ensure dilation in each dimension. We then define $q=|\operatorname{det}(\mathbf{A})|$ as the dilation factor.

Given a dilation matrix $\mathbf{A}$, a multiresolution analysis of $L_{2}\left(\mathbb{R}^{2}\right)$ on the hexagonal lattice with scaling function $\varphi$ is an increasing sequence $\cdots \subset V_{-1} \subset V_{0} \subset V_{1} \subset \cdots$ of subspaces of $L_{2}\left(\mathbb{R}^{2}\right)$ satisfying the four conditions

(i) (density) $\bigcup_{j \in \mathbb{Z}} V_{j}$ is dense in $L_{2}\left(\mathbb{R}^{2}\right)$;

(ii) (separation) $\bigcap_{j \in \mathbb{Z}} V_{j}=\{0\}$;

(iii) (scaling) $V_{j}=\left\{f\left(\mathbf{A}^{j} \mathbf{x}\right) \mid f(\mathbf{x}) \in V_{0}\right\} \quad \forall j \in \mathbb{Z}$;

(iv) $\left\{\varphi\left(\mathbf{x}-\mathbf{R}_{\text {hex }} \mathbf{k}\right) \mid \mathbf{k} \in \mathbb{Z}^{2}\right\}$ forms a Riesz basis of $V_{0}$;

In the next section, we design such multi-resolution analyses, with functions having the appealing property of rotation covariance. Before this, we introduce some more notions for manipulating signals on lattices. 
Let us consider a discrete signal $v=(v[\mathbf{k}])_{\mathbf{k} \in \mathbb{Z}^{2}}$ sampled on the hexagonal lattice $\Lambda_{\text {hex }}$; i.e., $v[\mathbf{k}]$ is located at the lattice site $\mathbf{R}_{\text {hex }} \mathbf{k}$. The discrete Fourier transform of $v$ is defined accordingly as

$$
\hat{v}(\boldsymbol{\omega})=\sum_{\mathbf{k} \in \mathbb{Z}^{2}} v[\mathbf{k}] \exp \left(-i\left\langle\boldsymbol{\omega}, \mathbf{R}_{\mathrm{hex}} \mathbf{k}\right\rangle\right)
$$

$\hat{v}(\boldsymbol{\omega})$ is periodic on the reciprocal lattice $\hat{\Lambda}_{\text {hex }}=2 \pi \widehat{\mathbf{R}}_{\text {hex }} \mathbb{Z}^{2}$, where $\widehat{\mathbf{R}}_{\text {hex }}=\mathbf{R}_{\text {hex }}^{-\mathrm{T}}$.

We define the cosets of $\Lambda_{\text {hex }}$ with respect to a dilation matrix $\mathbf{A}$ as the sets $\mathbf{A} \Lambda_{\text {hex }}+\boldsymbol{\tau}_{m}$, for $m=0, \ldots, q-1$, where $\boldsymbol{\tau}_{0}=\mathbf{0}$ and the vectors $\boldsymbol{\tau}_{m} \in \Lambda_{\text {hex }}$, called digits, are such that

$$
\Lambda_{\mathrm{hex}}=\bigcup_{m=0}^{q-1} \mathbf{A} \Lambda_{\mathrm{hex}}+\boldsymbol{\tau}_{m}
$$

A systematic way for choosing the digits is to take them as the lattice sites contained in the domain $\left\{\mathbf{A R}_{\text {hex }} \mathbf{x} \mid \mathbf{x} \in\right.$ $\left.[0,1)^{2}\right\}$ of $\mathbb{R}^{2}$. We also define the dual digits $\hat{\boldsymbol{\tau}}_{m}$ such that

$$
\widehat{\mathbf{A} \Lambda}_{\mathrm{hex}}=\mathbf{A}^{-\mathrm{T}} \hat{\Lambda}_{\mathrm{hex}}=\bigcup_{m=0}^{q-1} \hat{\Lambda}_{\mathrm{hex}}+\hat{\boldsymbol{\tau}}_{m}
$$

The upsampling operation of a discrete signal from $\Lambda_{\text {hex }}$ to $\mathbf{A} \Lambda_{\text {hex }}$ is defined as: ${ }^{16}$

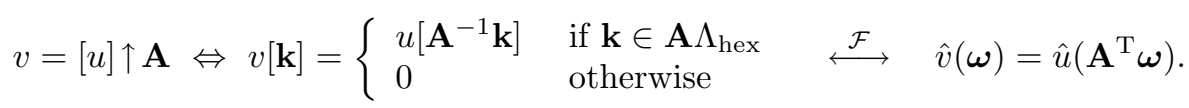

The downsampling operation of a discrete signal on $\Lambda_{\text {hex }}$ with matrix $\mathbf{A}$ is defined by: ${ }^{16}$

$$
v=[u] \downarrow \mathbf{A} \Leftrightarrow v[\mathbf{k}]=u[\mathbf{A k}] \quad \forall \mathbf{k} \in \mathbb{Z}^{2} \stackrel{\mathcal{F}}{\longleftrightarrow} \hat{v}(\boldsymbol{\omega})=\frac{1}{q} \sum_{m=0}^{q-1} \hat{u}\left(\mathbf{A}^{-\mathrm{T}} \boldsymbol{\omega}-\hat{\boldsymbol{\tau}}_{m}\right) .
$$

It is well known that applying a DWT to an image is equivalent to feeding it in a filterbank, using convolutions and the downsampling/upsampling operations defined above.

\section{LOCALIZED ROTATION COVARIANT FUNCTIONS}

A function $\rho(\mathbf{x})$ is isotropic if $\rho\left(\mathbf{R}_{\theta} \mathbf{x}\right)=\rho(\mathbf{x})$ for every $\mathbf{x} \in \mathbb{R}^{2}$, where $\mathbf{R}_{\theta}$ is the $2 \times 2$ rotation matrix with angle $\theta$. On the other hand, $\rho(\mathbf{x})$ is rotation-covariant if

$$
\rho\left(\mathbf{R}_{\theta} \mathbf{x}\right)=e^{i N \theta} \rho(\mathbf{x}), \quad \forall \mathbf{x} \in \mathbb{R}^{2}, \theta \in \mathbb{R},
$$

for some parameter $N \in \mathbb{Z}$. Thus, a rotation-covariant function has an isotropic magnitude and a phase that encodes the rotation of the analyzed function: by denoting $\mathbf{R}_{\theta} f=f\left(\mathbf{R}_{\theta} \cdot\right)$, we have:

$$
\left\langle\mathbf{R}_{\theta} f, \rho\right\rangle=\left\langle f, \mathbf{R}_{-\theta} \rho\right\rangle=e^{i N \theta}\langle f, \rho\rangle .
$$

The key property due to rotation-covariance is that the phase of the wavelet coefficients is related to some privileged local orientation, like the orientation of edges.

The starting point for the construction of our multiresolution bases is the following rotation-covariant function, defined in the sense of distributions by

$$
\hat{\rho}(\boldsymbol{\omega})=\frac{1}{\left(\omega_{1}^{2}+\omega_{2}^{2}\right)^{\alpha}\left(\omega_{1}-i \omega_{2}\right)^{N}},
$$

where $\boldsymbol{\omega}=\left[\omega_{1}, \omega_{2}\right]^{\mathrm{T}} \in \mathbb{R}^{2} \backslash \mathbf{0}, \alpha \in \mathbb{R}^{+}, N \in \mathbb{N}$, and the continuous Fourier transform of a bivariate function $f(\mathbf{x}) \in L_{2}\left(\mathbb{R}^{2}\right)$ is defined as $\hat{f}(\boldsymbol{\omega})=\int_{\mathbb{R}^{2}} f(\mathbf{x}) \exp (-i\langle\boldsymbol{\omega}, \mathbf{x}\rangle) d \mathbf{x}$, for $\boldsymbol{\omega} \in \mathbb{R}^{2}$. 
In the spatial domain, with polar coordinates $\mathbf{x}=[\|\mathbf{x}\| \cos (\theta),\|\mathbf{x}\| \sin (\theta)]^{\mathrm{T}}, \rho$ is defined as the inverse Fourier transform of the Hadamard partie finie $\operatorname{Pf}(\hat{\rho})$. This yields $\rho(\mathbf{x})=\|\mathbf{x}\|^{2 \alpha+N-2} e^{i N \theta} C(\alpha, N)$, where $\theta=\arg (\mathbf{x})$ and $C(\alpha, N)$ is a constant with eventually a $\ln (\|\mathbf{x}\|)$ factor. One clearly sees the rotation-covariance of $\rho$.

The translates of $\rho$ on $\Lambda_{\text {hex }}$ do not form a Riesz basis, so we have to localize this function and eliminate its singularity at the origin. For this, we define the elementary localization filter $\nu$ by

$$
\begin{aligned}
& \begin{array}{ll}
-1 & -1
\end{array} \\
& \nu=\frac{1}{\sqrt{3}} \begin{array}{cccc}
-1 & 6 & -1 \\
& -1 & -1
\end{array} .
\end{aligned}
$$

Equivalently, $\hat{\nu}(\boldsymbol{\omega})=\left(6-2 \cos \left(3^{1 / 4}\left(-\omega_{1} / \sqrt{3}+\omega_{2}\right) / \sqrt{2}\right)-2 \cos \left(3^{1 / 4}\left(\omega_{1} / \sqrt{3}+\omega_{2}\right) / \sqrt{2}\right)-2 \cos \left(3^{-1 / 4} \sqrt{2} \omega_{1}\right)\right) / \sqrt{3}$.

Then, we define the elementary polyharmonic hexagonal rotation-covariant (EPHRC) B-spline $\beta(\mathbf{x})$ as

$$
\beta(\mathbf{x})=\sum v^{\alpha+N / 2}[\mathbf{k}] \rho\left(\mathbf{x}-\mathbf{R}_{\mathrm{hex}} \mathbf{k}\right) \longleftrightarrow \hat{\beta}(\boldsymbol{\omega})=\frac{\hat{\nu}(\boldsymbol{\omega})^{\alpha+N / 2}}{\left(\omega_{1}^{2}+\omega_{2}^{2}\right)^{\alpha}\left(\omega_{1}-i \omega_{2}\right)^{N}} .
$$

Since $\hat{\nu}(\boldsymbol{\omega})=O\left(\|\boldsymbol{\omega}\|^{2}\right)$, the singularity of $\hat{\rho}$ at the origin is eliminated, and $\hat{\beta}(\boldsymbol{\omega}) \rightarrow 1$ as $\boldsymbol{\omega} \rightarrow \mathbf{0}$.

\subsection{The isotropic case $N=0$}

When $N=0, \beta$ is real-valued. In this case, our B-splines revert to classical polyharmonic splines, and $\beta$ is the hexagonal equivalent of the well-known elementary polyharmonic B-spline for the Cartesian lattice, defined by Rabut. ${ }^{17} \beta$ and its translates on $\Lambda_{\text {hex }}$ can be used, e.g., for interpolation on the hexagonal lattice, or for designing real DWTs on $\Lambda_{\text {hex }}$.

Our B-splines have better isotropy properties as their Cartesian counterparts of Rabut. Contrary to the latter, they converge to a Gaussian as the order $\alpha$ increases. More precisely, we have

$$
\hat{\beta}\left(\frac{\boldsymbol{\omega}}{\sqrt{\alpha}}\right)=1-\frac{1}{8 \sqrt{3}}\|\boldsymbol{\omega}\|^{2}+O\left(\|\boldsymbol{\omega}\|^{4}\right), \quad \text { as } \boldsymbol{\omega} \rightarrow \mathbf{0} .
$$

This means that the second-order moment of $\beta$ is $\alpha /(4 \sqrt{3})$. Then, following the outline of the proof of the central limit theorem, we get

$$
\hat{\beta}\left(\frac{\omega}{\sqrt{\alpha}}\right) \longrightarrow \exp \left(-\frac{1}{8 \sqrt{3}}\|\boldsymbol{\omega}\|^{2}\right), \quad \text { as } \alpha \rightarrow \infty,
$$

and $\beta$ tends to an isotropic Gaussian as $\alpha$ increases:

$$
\beta(\mathbf{x}) \approx \frac{2 \sqrt{3}}{\pi \alpha} \exp \left(-\frac{2 \sqrt{3}}{\alpha}\|\mathbf{x}\|^{2}\right),
$$

with standard deviation $\sqrt{\alpha /(4 \sqrt{3})}$. Thus, $\beta$ is bell-shaped with a width that can be tuned by $\alpha$. The convergence to a Gaussian indicates that $\beta$ tends to be optimally localized in both space and frequency, in the sense of the Heisenberg uncertainty principle.

In the following, we assume $N \geq 1$, so as to obtain the desired property of rotation-covariance.

\subsection{Key properties of the EPHRC B-splines}

Our B-splines $\beta$ have the following properties:

- Contrary to the function $\rho, \beta$ is not exactly rotation covariant. Actually, this property is incompatible with the requirement of having a Riesz basis and a positive approximation order at the same time. However, $\hat{\beta}$ is rotation-covariant of order 3 around the origin; that is,

$$
\left.\hat{\beta}(\boldsymbol{\omega})=e^{i N \theta}\left(1+C\|\boldsymbol{\omega}\|^{2}+R(\cos (6 \theta))\right)\|\boldsymbol{\omega}\|^{4}+O\left(\|\boldsymbol{\omega}\|^{6}\right)\right), \quad \text { as } \boldsymbol{\omega} \rightarrow \mathbf{0},
$$




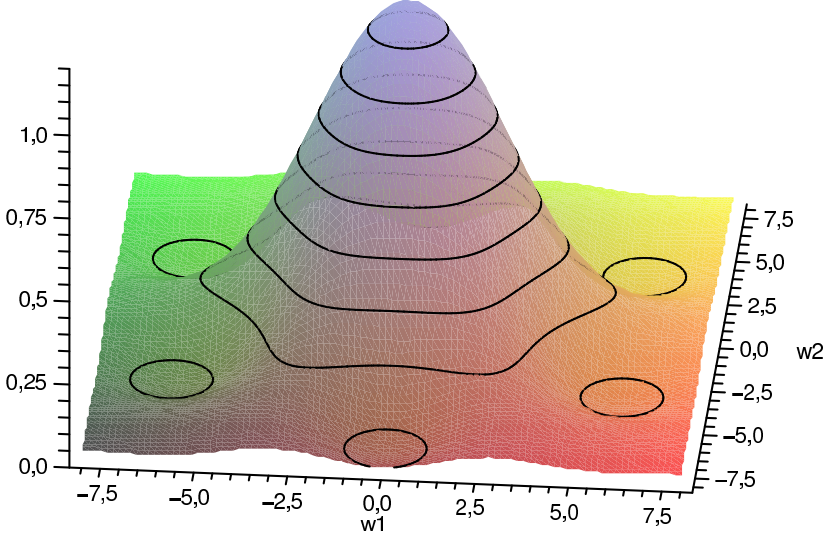

(a)

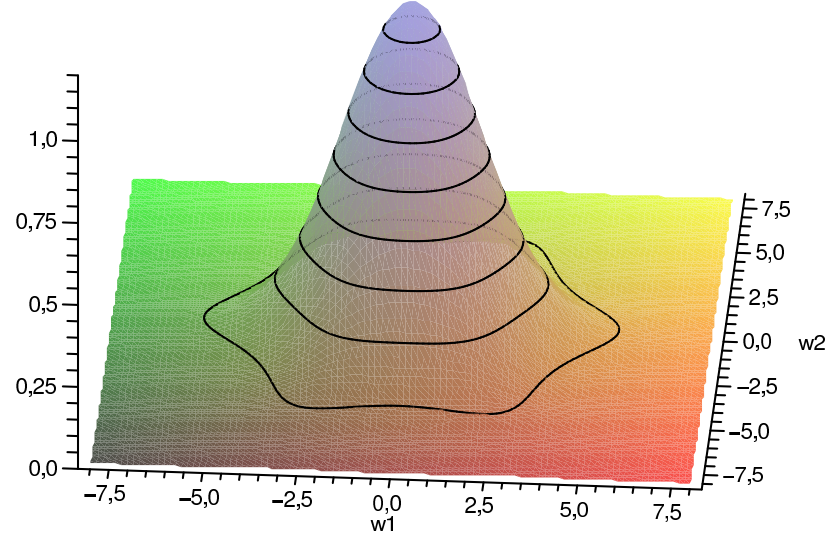

(b)

Figure 1. Plots of $|\hat{\beta}(\boldsymbol{\omega})|$, the magnitude of The Fourier transform of the EPHRC B-spline, for (a) $\alpha+N / 2=1$ and (b) $\alpha+N / 2=2$.

where $\theta=\arg (\boldsymbol{\omega}), C$ is a constant and $R(\cdot)$ is a non-constant function. With the additional 12 -fold symmetry of $\|\hat{\beta}\|$ and $\|\beta\|$, the rotation-covariance of $\beta$ is achieved to a good extent.

- As in the case $N=0$, we can show that the B-splines have a magnitude that tends to a Gaussian with variance $(2 \alpha+N) /(8 \sqrt{3})$. This property shows the good isotropy of the magnitude of $\beta$. An illustration is given in Fig. 1.

- The translates on the hexagonal lattice $\beta\left(\mathbf{x}-\mathbf{R}_{\mathrm{hex}} \mathbf{k}\right)$ generate a Riesz basis; i.e, there exist two constants $0<C_{0}, C_{1}<\infty$ such that

$$
C_{0}\|c\|_{\ell_{2}} \leq\left\|\sum_{\mathbf{k} \in \mathbb{Z}^{2}} c[\mathbf{k}] \beta\left(\mathbf{x}-\mathbf{R}_{\mathrm{hex}} \mathbf{k}\right)\right\|_{L_{2}} \leq C_{1}\|c\|_{\ell_{2}} .
$$

for every sequence $c \in \ell_{2}$. This condition is equivalent to

$$
C_{0} \leq \hat{a}_{\beta}(\omega) \leq C_{1}
$$

where we define the discrete autocorrelation sequence $a_{\beta}$ by $a_{\beta}[\mathbf{k}]=\left(\bar{\beta} * \beta^{*}\right)\left(\mathbf{R}_{\mathrm{hex}} \mathbf{k}\right)$, using the flip operator $\bar{\beta}(\mathbf{x})=\beta(-\mathbf{x})$ and the complex conjugation $\cdot^{*}$. The Poisson sum formula gives

$$
\hat{a}_{\beta}(\boldsymbol{\omega})=\sum_{\mathbf{k} \in \mathbb{Z}^{2}} a_{\beta}[\mathbf{k}] \exp \left(-i\left\langle\boldsymbol{\omega}, \mathbf{R}_{\mathrm{hex}} \mathbf{k}\right\rangle\right)=\sum_{\mathbf{k} \in \mathbb{Z}^{2}}\left|\hat{\beta}\left(\boldsymbol{\omega}-2 \pi \widehat{\mathbf{R}}_{\mathrm{hex}} \mathbf{k}\right)\right|^{2}
$$

Thus, given that $\hat{a}_{\beta}(\boldsymbol{\omega}) \geq|\hat{\beta}(\boldsymbol{\omega})|^{2}$ and that $|\hat{\beta}(\boldsymbol{\omega})|^{2}$ does not vanish in the Nyquist band, the existence of a lower bound $C_{0}$ is trivial. The existence of an upper bound $C_{1}$ can be derived as in Ref. 2, Appendix I.

- We can show that $\beta$ has an approximation order equal to $2 \alpha+N$.

\section{MULTIRESOLUTION BASES}

For every dilation matrix $\mathbf{A}$ that is a scaled rotation (that is, $\mathbf{A}=\sqrt{q} \mathbf{R}_{\vartheta}$ for some dilation factor $q \in \mathbb{R}^{2}$ and angle $\vartheta \in \mathbb{R}), \beta(\mathbf{x})$ satisfies a refinement relation with $\mathbf{A}$ :

$$
\beta\left(\mathbf{A}^{-1} \mathbf{x}\right)=\sum_{\mathbf{k} \in \mathbb{Z}^{2}} h_{\beta}[\mathbf{k}] \beta\left(\mathbf{x}-\mathbf{R}_{\mathrm{hex}} \mathbf{k}\right)
$$

and thus generates a multiresolution analysis. The associated refinement filter has the form:

$$
\hat{h}_{\beta}(\boldsymbol{\omega})=q \frac{\hat{\beta}\left(\mathbf{A}^{\mathrm{T}} \boldsymbol{\omega}\right)}{\hat{\beta}(\boldsymbol{\omega})}=\left(\frac{\hat{\nu}\left(\mathbf{A}^{\mathrm{T}} \boldsymbol{\omega}\right)}{\hat{\nu}(\boldsymbol{\omega})}\right)^{\alpha+N / 2} \frac{e^{-i N \vartheta}}{q^{\alpha+N / 2-1}} .
$$


Of particular interest is the dilation matrix $\mathbf{A}_{3}=\sqrt{3} \mathbf{R}_{\pi / 6}$. The corresponding dilation factor is $q=3$, which is the minimum value achievable on the hexagonal lattice with a dilation matrix corresponding to a scaled rotation.

We then define the polyharmonic hexagonal rotation-covariant spline space $V_{j}$ at scale $j \in \mathbb{Z}$ by

$$
V_{j}=\overline{\operatorname{Span}\left(\beta\left(\mathbf{A}^{j} \mathbf{x}-\mathbf{R}_{\text {hex }} \mathbf{k}\right) \mid \mathbf{k} \in \mathbb{Z}^{2}\right)}{ }^{L_{2}\left(\mathbb{R}^{2}\right)} .
$$

We also define the dual EPHRC B-spline $\beta^{d}$ as

$$
\hat{\beta}^{d}(\boldsymbol{\omega})=\frac{\hat{\beta}(\boldsymbol{\omega})}{\hat{a}_{\beta}(\boldsymbol{\omega})}
$$

and the orthonormal EPHRC B-spline $\beta^{\perp}$ as

$$
\hat{\beta}^{\perp}(\boldsymbol{\omega})=\frac{\hat{\beta}(\boldsymbol{\omega})}{\sqrt{\hat{a}_{\beta}(\boldsymbol{\omega})}} .
$$

In fact, with this definition, we have, for every $\mathbf{k} \in \mathbb{Z}^{2}$,

$$
\left\langle\beta^{\perp}(\mathbf{x}), \beta^{\perp}\left(\mathbf{x}-\mathbf{R}_{\text {hex }} \mathbf{k}\right)\right\rangle=\left\langle\beta(\mathbf{x}), \beta^{d}\left(\mathbf{x}-\mathbf{R}_{\text {hex }} \mathbf{k}\right)\right\rangle=\delta_{\mathbf{k}, \mathbf{0}},
$$

and the orthogonal projection of a function $f(\mathbf{x}) \in L_{2}$ onto $V_{0}$ can be written as

$$
\begin{aligned}
\mathcal{P}^{\perp} f(\mathbf{x}) & =\sum_{\mathbf{k} \in \mathbb{Z}^{2}}\left\langle f, \beta^{d}(\cdot-\mathbf{R} \mathbf{k})\right\rangle \beta\left(\mathbf{x}-\mathbf{R}_{\mathrm{hex}} \mathbf{k}\right) \\
& =\sum_{\mathbf{k} \in \mathbb{Z}^{2}}\left\langle f, \beta\left(\cdot-\mathbf{R}_{\mathrm{hex}} \mathbf{k}\right)\right\rangle \beta^{d}\left(\mathbf{x}-\mathbf{R}_{\mathrm{hex}} \mathbf{k}\right) \\
& =\sum_{\mathbf{k} \in \mathbb{Z}^{2}}\left\langle f, \beta^{\perp}\left(\cdot-\mathbf{R}_{\mathrm{hex}} \mathbf{k}\right)\right\rangle \beta^{\perp}\left(\mathbf{x}-\mathbf{R}_{\mathrm{hex}} \mathbf{k}\right) .
\end{aligned}
$$

\section{WAVELETS}

We can now construct polyharmonic hexagonal B-spline (pre-)wavelets that span the orthogonal complement $W_{j}$ of $V_{j}$ into $V_{j+1}$. It is known that $q-1$ wavelets $\psi_{m}(\mathbf{x})$ have to be designed for this, such that

$$
W_{j}=\overline{\operatorname{Span}\left(\psi_{m}\left(\mathbf{A}^{j} \mathbf{X}-\mathbf{R}_{\text {hex }} \mathbf{k}\right) \mid \mathbf{k} \in \mathbb{Z}^{2}, m=1 \ldots q-1\right)}{ }^{L_{2}\left(\mathbb{R}^{2}\right)} .
$$

The semi-orthogonality condition $W_{0} \perp V_{0}$ yields

$$
\left.\left\langle\psi_{m}(\mathbf{x}), \beta\left(\mathbf{x}-\mathbf{R}_{\text {hex }} \mathbf{k}\right)\right)\right\rangle=0,
$$

for every $\mathbf{k} \in \mathbb{Z}^{2}$ and $m=1 \ldots q-1$.

We define the high-pass filter $g_{\psi_{m}}$ associated to $\psi_{m}$, such that

$$
\psi_{m}\left(\mathbf{A}^{-1} \mathbf{x}\right)=\sum_{\mathbf{k} \in \mathbb{Z}^{2}} g_{\psi_{m}}[\mathbf{k}] \beta\left(\mathbf{x}-\mathbf{R}_{\mathrm{hex}} \mathbf{k}\right) \Leftrightarrow \quad \hat{g}_{\psi_{m}}(\boldsymbol{\omega})=q \frac{\hat{\psi}_{m}\left(\mathbf{A}^{\mathrm{T}} \boldsymbol{\omega}\right)}{\hat{\beta}(\boldsymbol{\omega})} .
$$

Thus, (29) is equivalent to

$$
\left[g_{\psi_{m}} * \bar{h}_{\beta}^{*} * a_{\beta}\right] \downarrow \mathbf{A}=0 .
$$

Traditionally, the wavelets are defined indirectly through (30), and the design is focused on the filters $g_{\psi_{m}}$ themselves. In the multivariate and non-separable case, this construction is not trivial. A method based on 
unitary polyphases matrices can be used, ${ }^{8,19}$ but it is practical only when the filters have finite impulse responses. In this work, we define the wavelets directly, in the Fourier domain:

$$
q \hat{\psi}_{m}\left(\mathbf{A}^{\mathrm{T}} \boldsymbol{\omega}\right)=\frac{\hat{\nu}(\boldsymbol{\omega})^{\alpha+N / 2}}{\hat{a}_{\beta}(\boldsymbol{\omega})} e^{-i\left\langle\boldsymbol{\omega}, \boldsymbol{\tau}_{m}\right\rangle} \hat{\beta}(\boldsymbol{\omega}) .
$$

We directly have the highpass filter

$$
\hat{g}_{\psi_{m}}(\boldsymbol{\omega})=\frac{\hat{\nu}(\boldsymbol{\omega})^{\alpha+N / 2}}{\hat{a}_{\beta}(\boldsymbol{\omega})} e^{-i\left\langle\boldsymbol{\omega}, \boldsymbol{\tau}_{m}\right\rangle}
$$

Let us prove that, with this definition, (31) holds. For this, we remark that for any filter $h_{1}$ such that $\hat{h}_{1}$

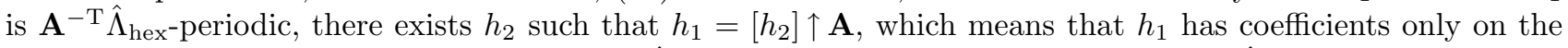
coset $\mathbf{A} \Lambda_{\text {hex }}$. As a consequence, for every $m,\left[\hat{h}_{1}(\boldsymbol{\omega}) e^{-i\left\langle\boldsymbol{\omega}, \boldsymbol{\tau}_{m}\right\rangle}\right] \downarrow \mathbf{A}=0$. Here, the role of $\hat{h}_{1}$ is played by

$$
\left(\frac{\hat{\nu}(\boldsymbol{\omega})^{\alpha+N / 2}}{\hat{a}_{\beta}(\boldsymbol{\omega})}\right) \hat{h}_{\beta}(\boldsymbol{\omega})^{*} \hat{a}_{\beta}(\boldsymbol{\omega})=\hat{\nu}\left(\mathbf{A}^{\mathrm{T}} \boldsymbol{\omega}\right)^{\alpha+N / 2} \frac{e^{i N \vartheta}}{q^{\alpha+N / 2-1}}
$$

which is clearly $\mathbf{A}^{-\mathrm{T}} \hat{\Lambda}_{\text {hex }}$-periodic. (31) follows. This proves the semi-orthogonality.

Let us now study the rotation-covariance properties of the wavelets. First, $\hat{\psi}_{m}$ has a rotation-covariant behavior around the origin:

$$
\hat{\psi}_{m}(\boldsymbol{\omega})=O\left(\|\boldsymbol{\omega}\|^{2 \alpha+N} \hat{\beta}(\boldsymbol{\omega})\right)=O\left(\|\boldsymbol{\omega}\|^{2 \alpha+N} e^{i N \theta}\right)
$$

Moreover, the wavelet transform of a test function $f$,

$$
\begin{aligned}
\left\langle f, \psi_{m}\left(\cdot-\mathbf{R}_{\mathrm{hex}} \mathbf{k}\right)\right\rangle & =\frac{1}{(2 \pi)^{2}}\left\langle\hat{f}, \psi_{m}\left(\widehat{-\mathbf{R}_{\mathrm{hex}}} \mathbf{k}\right)\right\rangle \\
& =\frac{1}{(2 \pi)^{2}} \int_{\mathbb{R}^{2}} \hat{f}(\boldsymbol{\omega}) \hat{\psi}_{m}(\boldsymbol{\omega})^{*} e^{i\left\langle\boldsymbol{\omega}, \mathbf{R}_{\mathrm{hex}} \mathbf{k}\right\rangle} d \boldsymbol{\omega} \\
& =\frac{1}{(2 \pi)^{2}} \int_{\mathbb{R}^{2}} \hat{f}(\boldsymbol{\omega})\|\boldsymbol{\omega}\|^{2 \alpha}\left(\omega_{1}-i \omega_{2}\right)^{N} \hat{\Phi}(\boldsymbol{\omega}) e^{i\left\langle\boldsymbol{\omega}, \mathbf{A}^{-1} \boldsymbol{\tau}_{m}\right\rangle} e^{i\left\langle\boldsymbol{\omega}, \mathbf{R}_{\mathrm{hex}} \mathbf{k}\right\rangle} d \boldsymbol{\omega} \\
& =(-\Delta)^{\alpha}\left(-i \frac{\partial}{\partial x_{1}}-\frac{\partial}{\partial x_{2}}\right)^{N}\{f * \Phi\}\left(\mathbf{R}_{\mathrm{hex}} \mathbf{k}+\mathbf{A}^{-1} \tau_{m}\right),
\end{aligned}
$$

behaves as a Laplacian of order $\alpha$ modified by Wirtinger differential operator of order $N$. Here, $\Phi(\mathbf{x})$ is a lowpass, 12 -fold symmetric, and isotropic around the origin, kernel, given by

$$
q \hat{\Phi}\left(\mathbf{A}^{\mathrm{T}} \boldsymbol{\omega}\right)=\frac{e^{-i N \vartheta}}{q^{\alpha+N / 2}} \frac{\hat{\nu}(\boldsymbol{\omega})^{2 \alpha+N}}{\|\boldsymbol{\omega}\|^{4 \alpha+2 N} \hat{a}_{\beta}(\boldsymbol{\omega})} \quad \stackrel{\mathcal{F}}{\longleftrightarrow} \Phi\left(\mathbf{A}^{-1} \mathbf{x}\right)=\frac{e^{-i N \vartheta}}{q^{\alpha+N / 2}} \beta(\mathbf{x}) * \bar{\beta}^{d}(\mathbf{x})^{*}
$$

Thus, the wavelets can be represented as

$$
\psi_{m}(\mathbf{x})=(-\Delta)^{\alpha}\left(-i \frac{\partial}{\partial x_{1}}+\frac{\partial}{\partial x_{2}}\right)^{N}\left\{\Phi^{*}\right\}\left(\mathbf{x}-\mathbf{A}^{-1} \tau_{m}\right) .
$$

This last expression clearly shows the main feature of our wavelets: when $N=1$, analyzing a function $f$ with the wavelets extracts the direction of the gradient of $f$ in the phase of the wavelet coefficients. 


\section{IMPLEMENTATION}

Because the filters used for the wavelet decomposition have infinite impulse responses and are not separable, an implementation by convolutions in space domain is not suitable: this would be costly and would yield to accuracy problems because of the necessity to truncate the filters.

Since the filters have explicit formulas in the Fourier domain, it is natural and more convenient to use FFTs for computing the wavelet decomposition in the Fourier domain. Thus, the discrete Fourier transform of the image data is filtered by multiplying with the refinement and the wavelet filter. Exploiting symmetries, we can downsample the data by a factor of three and use an inverse Fourier transform on the reduced data. This yields a fast and stable algorithm.

\section{CONCLUSION}

We presented a family of complex multiresolution bases in $L_{2}\left(\mathbf{R}^{2}\right)$ deployed on the hexagonal lattice. The nonseparable B-splines we introduced have the property of approximate rotation-covariance and yield multiresolution analyses for every scaled rotation dilation matrix. They are flexibly parameterized by the value $\alpha$ that controls the smoothness and decay properties of the resulting wavelets. The second degree of freedom, $N$, influences the rotation-covariance behavior. Both parameters can be important for image processing applications.

The wavelet transform is non-redundant when applied to complex-values signals. It is $2 \times$ redundant for real-valued images. The FFT-based implementation provides a fast algorithm, which makes our wavelet decomposition no more complicated to use than the classical real DWT.

\section{ACKNOWLEDGMENTS}

The first two authors are supported by the Marie Curie Excellence Team Grant MEXT-CT-2004-013477, Acronym MAMEBIA, funded by the European Commission. The last author was supported in part by the swiss National Science Fondation under Grant 200020-101821 and by the Center for Biomedical Imaging (CIBM) of the GenevaLausanne Universities and the EPFL, as well as the Fondations Leenaards and Louis-Jeantet.

The authors would like to thank Prof. Michael Unser for fruitful discussions on the construction of wavelets on arbitrary lattices.

\section{REFERENCES}

1. B. Han and R.-Q. Jia, "Quincunx fundamental refinable functions and quincunx biorthogonal wavelets," Math. Comput. 71, pp. 165-196, 2002.

2. D. Van De Ville, T. Blu, and M. Unser, "Isotropic polyharmonic B-splines: Scaling functions and wavelets," IEEE Trans. Image Processing 14, pp. 1798-1813, Nov. 2005.

3. R. M. Mersereau, "The processing of hexagonally sampled two-dimensional signals," Proc. IEEE 67, pp. 930949, June 1979.

4. L. Condat, B. Forster-Heinlein, and D. V. D. Ville, " $\mathrm{H}_{2} \mathrm{O}$ : reversible hexagonal-orthogonal grid conversion by 1-D filtering," in Proc. of IEEE ICIP, Sept. 2007. to appear.

5. S. Mallat, "A theory for multiresolution signal decomposition: The wavelet representation," IEEE Trans. Pattern Anal. Machine Intell. 11, pp. 674-693, July 1989.

6. J. Kovačević and W. Sweldens, "Wavelet families of increasing order in arbitrary dimensions," IEEE Trans. Image Processing 9, pp. 480-496, Mar. 2000.

7. R. S. Strichartz, "Wavelets and self-affine tilings," Constructive Approximation 9, pp. 327-346, 1993.

8. I. Daubechies, Ten lectures on wavelets, Society for Industrial and Applied Mathematics, Philadelphia, PA, USA, 1992.

9. B. Forster, T. Blu, and M. Unser, "A new family of complex rotation-covariant multiresolution bases in 2-D," in Proc. of the SPIE Conf. on Mathematical Imaging: Wavelet Applications in Signal and Image Proc. X, 5207, pp. 475-479, (San Diego CA, USA), Aug. 2003. Part I.

10. N. Kingsbury, "Complex wavelets for shift invariant analysis and filtering of signals," J. Appl. Comput. Harmon. Anal. 10, pp. 234-253, May 2001. 
11. I. W. Selesnick, "Hilbert transform pairs of wavelet bases," IEEE Signal Processing Lett. 8, pp. 170-173, June 2001.

12. F. C. A. Fernandes, R. L. C. van Spaendonck, and C. S. Burrus, "A new framework for complex wavelet transforms," IEEE Trans. Signal Processing 51, July 2003.

13. R. van Spaendonck, T. Blu, R. Baraniuk, and M. Vetterli, "Orthogonal hilbert transform filter banks and wavelets," in IEEE Proc. of ICASSP, 2003.

14. F. Fernandes, M. Wakin, and R. G. Baraniuk, "Non-redundant, linear-phase, semi-orthogonal, directional complex wavelets," in IEEE Proc. of ICASSP, 2, pp. 953-956, May 2004.

15. R. A. Ulichney, Digital Halftoning, MIT Press, 1987.

16. E. Viscito and J. P. Allebach, "The analysis and design of multidimensional fir perfect reconstruction filter banks for arbitrary sampling lattices," IEEE Trans. Circuits Syst. 38, pp. 29-41, Jan. 1981.

17. C. Rabut, "Elementary m-harmonic cardinal B-splines," Numer. Algorithms 2, pp. 39-62, 1992.

18. D. Van De Ville, T. Blu, M. Unser, W. Philips, I. Lemahieu, and R. Van De Walle, "Hex-spline: A novel family for hexagonal lattices," IEEE Trans. Image Processing 13, pp. 758-772, June 2004.

19. P. Wojtaszczyk, A Mathematical Introduction to Wavelets, vol. 37, Cambridge University Press, Cambridge, 1997. 